\title{
Anaesthesia Related Death in Prader Willi Syndrome: Appropriate Management and Medical Liability
}

\author{
Snenghi $\mathbf{R}^{1 *}$, El Mazloum $\mathbf{R}^{1}$, Montisci $\mathbf{M}^{1}$, \\ Feltracco $\mathrm{P}^{2}$ and Barbieri $\mathrm{S}^{2}$ \\ ${ }^{1}$ Department of Cardiac, Thoracic and Vascular Sciences, \\ University of Padova, Italy \\ ${ }^{2}$ Department of Anesthesia, University of Padova, Italy \\ *Corresponding author: Dott.ssa Rossella Snenghi, \\ Department of Cardiac, Thoracic and Vascular Sciences, \\ University of Padova, Italy
}

Received: March 14, 2017; Accepted: March 30, 2017; Published: April 05, 2017

\begin{abstract}
Prader - Willi syndrome (PWS) is a complex multisystem genetic disorder associated with a constant chromosomal abnormality. The anesthesiological care of patients with PWS include a careful approach to the potential difficulties with airway, an intraoperative strict control of ventilatory modality, proper management of metabolic disturbances and adequate cardio circulatory support.

The PWS requires various heterogeneous surgical interventions such as squint correction, spinal surgery for scoliosis, palatal and orthopedic surgery, dental rehabilitation for common tooth decay, cholecystectomy, pacemaker placement and orchidopexy for cryptorchidism

For these reasons patients with PWS may frequently undergo local, regional and general anesthesia. Some Authors describe data related to patients with medical complex disease to have a higher rate of mortality and morbidity and poor outcome which reported for procedures performed in and outside the operative room.
\end{abstract}

We present a fatal case of severe bronchospasm with serious hypoxia during general anesthesia in a young patient affected by PWS with chronic pancreatitis and biliary calculosis.

\section{Introduction}

Prader - Willy syndrome (PWS) is a complex multisystem genetic disorder in which some genes (or subsets) on chromosome 15 are deleted or unexpressed. There are 3 main genetic subtypes in PWS: paternal 15q11-q13 deletion (65-75\% of cases), maternal uniparental disomy of chromosome 15 (20-30\% of cases) and imprinting defect $(1-3 \%)$

$[1,2]$. Clinical course can be divided into infancy, the early phase of the condition, characterized by hypotonia, lethargy and hypoventilation associated with aspiration and unexpected death; the following phase, childhood (at 2-4 years of age), which is characterized by hyperphagia and subsequently obesity [3]. In the childhood and adolescent period the characteristic behaviour and cognitive problems are associated with strabismus, dysmorphic facial appearance, scoliosis, temperature regulatory abnormalities, respiratory insufficiency (this aspect is particularly interesting in the intra and perioperative management of PWS patients). Other signs of this disorder are temperature deregulation, short stature, scoliosis, mild mental retardation, epilepsy and sleep apnoea [4-6].

For all ages, the annual death rate is $3 \%$, but for over 30 years of age, the death rate reaches $7 \%$. PWS patients generally die early from complications related to obesity, such as diabetes mellitus, cardiovascular disease and respiratory failure. Insulin resistance in the presence of proper insulin production is a characteristic of early stages while as the disease progresses the production of insulin becomes insufficient and it needs to be replaced. Hardened arteries, due to diabetes complications, hypertension, and elevated blood cholesterol levels contribute to a higher risk of coronary artery disease, stroke, malignant arrhythmias and sudden death. Cardiomegaly, corpylmonale and possible intraoperative shunting in adolescents and adults are other important causes of muscle weakness, severe dyspnoea on minimal exercise and rapid cardiopulmonary compromise. Sudden unexpected death can be also attributed to hypothalamic dysfunction which determines a poor ventilator response to hypoxia. Some Authors often reported sudden unexpected clinical course and deaths in infants and children caused by respiratory infections, hypoventilation and respiratory obstruction [7].

The PWS requires various heterogeneous surgical interventions such as squint correction, spinal surgery for scoliosis, palatal and orthopedic surgery, dental rehabilitation for common tooth decay, cholecystectomy, pacemaker placement, and orchidopexy for cryptorchidism [8]. For these reasons patients with PWS may frequently undergo local, regional and general anesthesia. Some Authors describe data related to patients with medical complex disease to have a higher rate of mortality and morbidity and poor outcome which reported for procedures performed in and outside the operative room.

The anesthesiological care of patients with PWS include a careful approach to the potential difficulties with airway, an intraoperative strict control of ventilatory modality, proper management of metabolic disturbances and adequate cardio circulatory support $[9,10]$.

Abnormalities in the central control of ventilation, associated with preoperative respiratory failure substantially increase the perioperative risk of regional and general anesthesia. In fact, severe oxygen desaturation and bronchospasm may occur both during
Austin J Anesthesia and Analgesia - Volume 5 Issue 1 - 2017 ISSN : 2381-893X | www.austinpublishing group.com Snenghi et al. ( All rights are reserved
Citation: Snenghi R, El Mazloum R, Montisci M, Feltracco P and Barbieri S. Anaesthesia Related Death in Prader Willi Syndrome: Appropriate Management and Medical Liability. Austin J Anesthesia and Analgesia. 2017; 5(1): 1053. 
anesthetic induction or in the intraoperative course and at the end of anesthesia. Multiple and complex causes make anesthesia management more difficult in PWS. The most frequently reported abnormalities in these patients are central nervous system disturbance associated with pulmonary restrictive defect due to spine scoliosis, abnormality of peripheral chemoreceptor pathways, sleep apnoea with desaturation, abnormally thick and viscous saliva, obesity and poor pharyngeal muscle tone, cardiovascular abnormalities, thermoregulatory dysfunction with or without acidosis [7,8,10-13] We present a fatal case of severe bronchospasm with serious hypoxia during general anesthesia in a young patient affected by PWS with chronic pancreatitis and biliary calculosis.

\section{Case Presentation}

A 17-year-old girl affected by PWS, based on clinical examination and referred genetic analysis, associated with obesity (body mass index- BMI - of $46,7 \mathrm{~kg} / \mathrm{m}^{2}$ ) and a medical history of biliary calculosis, was scheduled for elective cholecystectomy with intraoperative Endoscopic Retrograde Cholangiopancreatography (ERCP) under general anesthesia following 2 previous pancreatitis episodes. The patient had never experienced general anesthesia but only an unsuccessfully light sedation. Based on the recognized potential perioperative complications occurring when anesthesia is required for various surgical interventions, anaesthesiological approach included an accurate pre-operative evaluation and a careful pre-induction plan aimed at facilitating tracheal intubation. A thorough evaluation of past and current cardio circulatory status was undertaken, as well as history of defective central control of ventilation, obstructive/sleeps apnoea, obstructive respiratory disorders, gastro-oesophageal reflux, nocturnal aspiration, reduced salivary flow. Active coughing and thick bronchial secretions were absent and previous laryngospasm or bronchospasm episodes denied by her mother. Accurate inspection was performed to rule out the presence of micrognatia, mandibular hypoplasia, poor dentition, large tongue, palatal abnormalities, adenoid hypertrophy, and limited neck mobility. At preoperative anaesthesiological evaluation no major concerns emerged on potential difficult airway management. Yet, laryngeal mask, intubating stylets "small" tracheal tubes, video laryngoscope, a fiberoptic bronchoscope, and other appropriate equipment to deal with the "cannot intubate/cannot ventilate" scenario, were immediately available on anesthesia workstation; an efficient suction device put very close to the table in case of aspiration during induction of anesthesia. Sufficient preoperative fasting and premedication with oral pantoprazole were confirmed.

She arrived in OR (Operative Room) with tachycardia (120bpm) and pulse oximetry of $99 \%$ on room air, blood pressure of $150 / 90 \mathrm{mmHg}$, respiratory rate of 16 breaths/min. Mask ventilation was performed easily; tracheal intubation was not difficult; at mouth inspection she was classified as Mallampati class III and Cormack 1, despite the short neck and the characteristic facies. Foreign substances were not observed in the mouth during endotracheal intubation. For induction she was given Midazolam 1mg, Propofol 180mg, Fentanyl $100 \mathrm{mcg}$ and rocuronium $100 \mathrm{mg}$. Invasive continuous radial artery catheterization was applied in order to monitor hemodynamic status. The stomach was decompressed soon after intubation. Anaesthesia maintenance occurred with fentanyl and sevoflurane. Since the beginning of mechanical ventilation high inspiratory and plateau pressures were detected at respiratory monitoring despite the reduction of ideal tidal volume. Before the completion of the endoscopic procedure severe bronchospasm ensued, associated with high inflation pressures, marked hypoventilation, decrease in end-tidal $\mathrm{CO}_{2}$, and progressive air trapping. Chest auscultation first revealed diffuse and bilateral expiratory wheezing, and then almost no respiratory sounds on both lungs. Direct laryngoscopy and thereafter bronchoscopy confirmed the correct position of tracheal tube and excluded bronchus obstruction or aspiration of regurgitated gastric contents. In spite of adequate anesthetic depth, inhaled bronchodilator, xilocaine i.v. and sevoflurane at $3 \%$, no bronchodilation occurred, and the $\mathrm{SaO}_{2}$ dropped persistently down to $75-85 \%$. Manual ventilation of the patient showed "stiff lung" inflation, significant inspiratory stridor, with no apparent exhalation of inflated gas.

Severe hypotension $(60 / 30 \mathrm{mmHg})$ and bradycardia $(23 \mathrm{bpm})$ followed, and the patient was administered $2 \mathrm{mg}$ of atropine, $2 \mathrm{mg}$ of epinephrine, $100 \mathrm{mEq}$ bicarbonate and $15.000 \mathrm{U}$ of heparin. Transthoracic Echocardiography (TTE) excluded cardiac thromboembolism, pneumothorax, and revealed a markedly dilated right ventricle. In the following $15-20 \mathrm{~min}$ the severe bronchospasm gradually recovered with partial resolution of pulmonary stiffness and normalization of oxygen saturation. The patient was transferred to the intensive care, where pneumothorax, massive atelectasis, pulmonary emboli and myocardial infarction were excluded. After cessation of sedation the patient did not regain consciousness, spontaneous ventilation or evoked movements and after a week was declared death for cerebral death.

\section{Autopsy Evidence}

The external examination of the body revealed strong obesity with typical features of PWS (face, hands, feet), outcomes of surgical access at the abdomen and venous access in the upper limbs.

The autopsy showed the cerebral oedema, lung congestion, septal myocardial ischemia, pulmonary congestion with emphysema and oedema, hepatic congestion and autolysis of the pancreas with wax stains. Pulmonary embolism and acute abdomen such as pancreatitis was excluded. Lung histology was unspecific because the death occurred after a week in intensive care. However there was no significant evidence for chronic conditions. The organ weights are reported in Table 1 .

\section{Discussion}

The macro-microscopic frameworks evidenced at the autopsy identify as "causa mortis" the acute brain oedema. However, the death occurred too late compared to the generalized framework hypoxic-ischemic, so the autopsy did not allow to identify with certainty the aetiology of sudden desaturation. In this particular case we note that the weight of the lungs compared to lower BMI (25-40 vs 47 ) is abnormally low (right $320 \mathrm{~g}$ - left $240 \mathrm{gvs} 561 \pm 256-491 \pm 204$ ) [14]. Very likely an almost impossible tidal volume expiration due to the remarkable bronchoconstriction, and the subsequent "breath-stacking" caused by vigorous manual insufflations, lead to massive, irreversible air trapping. Abnormal inflation of small lungs was responsible for "pulmonary tamponade", severe increase in pulmonary vascular resistance, pulmonary hypoperfusion and 
Table 1: Organ weights (grams).

Table 1: Organ weights (grams).
\begin{tabular}{|c|c|c|}
\hline & Autopsy & $\begin{array}{c}\text { Mean 25-40 BMI range } \\
\text { (Lorin del la Grandmaison G et al, 2001) }\end{array}$ \\
\hline Heart & 330 & $362 \pm 77$ \\
\hline Right lung & 320 & $561 \pm 256$ \\
\hline Left lung & 240 & $491 \pm 204$ \\
\hline Liver & 1950 & $1609 \pm 419$ \\
\hline Spleen & 150 & $152 \pm 67$ \\
\hline Right kidney & 120 & $144 \pm 40$ \\
\hline Left kidney & 90 & $146 \pm 38$ \\
\hline
\end{tabular}

intolerable hypoxia, serious increase in right ventricular after load, right ventricle over distension, left displacement of interventricular septum, marked reduction in venous return, and frank cardiogenic shock. Nevertheless, the retrospective clinical analysis of the case allows to suggest the following considerations: 1) the appropriate steps were taken to resolve the bronchospasm crisis management; 2) the history excluded a pre-anesthetic history of asthma, chronic or acute bronchitis, reflux, heavy smoking or esophagitis; 3) the anesthetist monitored carefully the PWS patient; 4) the bronchospasm wasn't associated with difficult intubation that could have caused the irritation of the airway or laryngospasm; 5) the bronchospasm presumed due to airway irritation had no defined cause (did not occur following pulmonary oedema, anaphylaxis or severe allergy); 6) the first sign of alert (high inspiratory and plateau pressure) were detected, even though, the sudden unexpected severe bronchoconstriction was not preventable.

Generally, it is known that mortality incidence during anesthesia ranges from 0.5 to 1.0 per 10.000 anesthetics [15] or is about $3-4 \%$ in western medicine. Management of anatomic and functional airway problems (bronchospasm, laryngospasm, muscle rigidity, alveolar collapse) in pediatric and adult patients continues to result in significant morbidity and mortality [16]. Most deaths are due to underlying illness and frailty. Gouel-Cheron et al. reported in the work that a History of Asthma (HA) is associated with a higher risk of mortality of Anaphylactic Shock (AS), but it is unknown whether this association remains valid for intra-operative AS, the factor associated with bronchospasm was a neuromuscular blocking drug, with both IgE- or non-IgE-mediated reactions [17]. These results suggest that the mechanisms of bronchospasm in AS may be different from those of asthma and that, in the presence of bronchospasm during anesthesia, AS should be considered to be the most likely cause.

The causes of mortality in PWS described in literature are endocarditis, pulmonary thromboembolism, respiratory failure and sepsis [7]. The Italian National Survey for PWS reported that obesity related conditions were the cause of death in 9 of 12 individuals [18]. Surgical procedures in PWS are associated with adverse events; usually minor. The Committee on Genetics of American Academy of Pediatrics, stressed the necessity of medical therapy before surgery (i.e. for treatment of undescended testes) such as avoiding general anesthesia for infant with low muscle tone and potential underlying respiratory compromise $[19,20]$. The skills of the anesthetist and surgeon are an important prerequisite for a good outcome in operative rooms and a significant percentage of the complications can lead to serious injury or death. It is important to consider if anesthesia can contribute to perioperative mortality and if critical clinic aspects of PWS with preventive strategies may reduce the mortality rates in these individuals. Appropriate structured approach to these patients and management of the earlier recognition of the problems, the correct documentation in the anesthetic records, and prompt action can resolve the crisis and the complication that can occurred. It is clear that, although the patient was mechanically ventilated, the desaturation occurred during the final phase of the endoscopic procedure and this was due to acute obstructive/restrictive airway pathology; other conditions which may give sudden desaturation have been excluded. Moreover, other cases of laryngospasm with desaturation during some surgical procedure are described [21].

Furthermore, in PWS patients, sufficient consideration should be given when selecting medication. There are now a day's two common volatile agents for the maintenance of anesthesia, desflurane and sevoflurane; since they accumulate less in fat cells and patients recover quickly. Among those two, sevoflurane was selected because of the following effects: less irritation on the airways, bronchodilative effects and repression of hypoxic pulmonary vasoconstriction [22]. Both succinylcholine and non-depolarizing relaxants can be safely used in patients with PWS [23]. Additionally, in PWS patients, morbid obesity increases the abdominal pressure, which increases the occurrence rate of hiatal hernia along with the risk of aspiration pneumonia [24].

\section{Conclusion}

The case presented stresses the importance of an appropriate preoperative evaluation of patients with Prader-Willy syndrome, which should also include attention to trachea-bronchial reactivity. Lung function tests, chest X-ray images, and the preoperative assessment of lung mechanics, may be useful to rule out abnormalities in the upper and lower respiratory systems. Basal spirometry and the preoperative functional evaluation of airway reactivity may disclose unrecognized disorders of respiration quantify the degree of narrowing and "orient" to potential difficulties in intraoperative mechanical ventilation.

In our patient the bronchial reactivity tests (methacoholine or histamine challenge test) were not performed preoperatively and probably, the potential high individual intrinsic risk of bronchospasm following upper digestive tract and airway instrumentation remained undetected at preoperative assessment. Nevertheless, due to the frequent association of morbid obesity with difficult airway management, aspiration risk, and haemoglobin-oxygen desaturation, both a careful inspection of oral cavity and the appropriate equipment for a difficult intubation were provided before induction of anesthesia. Clear anatomic abnormalities, damaged teeth, thick sticky saliva, gastric content from potential vomiting or rumination, were excluded. A history of asthma was not detected, tracheal intubation was very smooth, mechanical ventilation "gently", nonetheless tremendous bronchospasm did occur, and even if it apparently resoled after $2 \mathrm{mg}$ epinephrine, bronchospasm-induced hypoxia persisted long enough to cause irreversible brain damage.

It has to be underlined that in these patients, even interventions under general anesthesia are not to be considered as safe they seem and the autopsy is not always able to give the answer to the cause of death. 


\section{References}

1. Rocha CF, Paiva CL. Prader-Willi-like phenotypes: a systematic review of their chromosomal abnormalities. Genet. Mol. Res. 2014; 13: 2290-2298.

2. Angulo MA, Butler MG, Cataletto ME. Prader-Willi syndrome: a review of clinical, genetic and endocrine findings. J. Endocrinol. Invest. 2015; 38: 1249 1263.

3. Mascari MJ, Gottlieb W, Rogan PK, et al. The frequency of uniparenta disomy in Prader-Willi syndrome. Implications for molecular diagnosis. N. Engl. J. Med. 1992; 326: 1599-1607.

4. Cassidy SB, Driscoll DJ. Prader-Willi syndrome. Eur. J. Hum. Genet. 2009 17: $3-13$

5. Yee BJ, Buchanan PR, Mahadev S, et al. Assessment of sleep and breathing in adults with prader-willi syndrome: a case control series. J. Clin. Sleep Med. 2007; 3: 713-718.

6. Bridges N. What is the value of growth hormone therapy in Prader Will syndrome? Arch. Dis. Child. 2014; 99: 166-170.

7. Nishikawa M, Mizutani T, Nakao T, et al. Respiratory failure due to morbid obesity in a patient with Prader-Willi syndrome: an experience of long-term mechanical ventilation. J. Anesth. 2006; 20: 300-303

8. Lionti T, Reid SM, Rowell MM. Prader-Willi syndrome in Victoria: mortality and causes of death. J. Paediatr. Child Health. 2012; 48: 506 -511.

9. Dearlove OR, Dobson A, Super M. Anaesthesia and Prader-Willi syndrome. Paediatr. Anaesth. 1998; 8: 267-271.

10. Kawahito $\mathrm{S}$, Kitahata $\mathrm{H}$, Kimura $\mathrm{H}$, et al. Bronchospasm during anesthesia in a patient with Prader-Willi syndrome. Masui. 1995; 44: 1675-1679.

11. Choi JW, Kim EJ, Min BW, et al. Experience of severe desaturation during anesthetic induction period in an obese adult patient with Prader-Will syndrome -A case report. Korean J. Anesthesiol. 2012; 62: 179-183.

12. Gozal D, Arens R, Omlin KJ, et al. Absent peripheral chemo sensitivity in Prader-Willi syndrome. J. Appl. Physiol. 1994; 77: 2231-2236.

13. Arens R, Gozal D, Omlin KJ, et al. Hypoxic and hypercapnic ventilatory responses in Prader-Willi syndrome. J. Appl. Physiol. 1994; 77: 2224-2230.
14. Arens R, Gozal D, Burrell BC, et al. Arousal and cardio respiratory responses to hypoxia in Prader-Willi syndrome. Am J Respir Crit Care Med. 1996; 153: 283-287.

15. De la Grandmaison GL, Clairand I, Durigon M. Organ weight in 684 adult autopsies: new tables for a Caucasoid population. Forensic Sci. Int. 2001 119: $149-154$.

16. Braz LG, Braz DG, Cruz DS, et al. Mortality in anesthesia: a systematic review. Clinics (Sao. Paulo). 2009; 64: 999-1006.

17. Engelhardt T, Weiss M. A child with a difficult airway: what do I do next? Curr. Opin. Anaesthesiol. 2012; 25: 326-332.

18. Gouel-Cheron, Neukirch C, Aubier B, et al. Anaphylactic bronchospasm during general anesthesia is not related to asthma. Allergy. 2015; 70: 453456

19. Grugni G, Crino A, Bosio L, et al. The Italian National Survey for PraderWilli syndrome: an epidemiologic study. Am. J. Med. Genet. A. 2008; 146: 861-872.

20. Cataletto M, Angulo M, Hertz G, Whitman B. Prader-Willi syndrome: A primer for clinicians. Int. J. Pediatr. Endocrinol. 2011; 1: 12.

21. Mc Candless SE, Committee on Genetics. Clinical report-health supervision for children with Prader-Willi syndrome. Pediatrics. 2011; 127: 195-204.

22. Anesthetic management of Prader-Willi syndrome: what if neuromuscular relaxants could not be avoided? - PubMed - NCBI 2015

23. Doi M, Takahashi T, Ikeda K. Respiratory effects of sevoflurane used in combination with nitrous oxide and surgical stimulation. J. Clin. Anesth. 1994; 6: 1-4.

24. Yamashita M, Koishi K, Yamaya R. et al. Anaesthetic considerations in the Prader-Willi syndrome: report of four cases. Can. Anaesth. Soc. J. 1983; 30 179-184.

25. Sloan TB, Kaye Cl. Rumination risk of aspiration of gastric contents in the Prader-Willi syndrome. Anesth. Analg. 1991; 73: 492-495.
Austin J Anesthesia and Analgesia - Volume 5 Issue 1 - 2017 ISSN : 2381-893X | www.austinpublishing group.com Snenghi et al. () All rights are reserved
Citation: Snenghi R, El Mazloum R, Montisci M, Feltracco P and Barbieri S. Anaesthesia Related Death in Prader Willi Syndrome: Appropriate Management and Medical Liability. Austin J Anesthesia and Analgesia. 2017; 5(1): 1053. 